\title{
Editorial
}

\section{Editorship at SWSC}

Jean Lilensten and Anna Belehaki

Space Weather is quite a new discipline. Awareness about Space Weather started to grow in the middle of the $20^{\text {th }}$ century (with, e.g., the first broadcasts of the "ursigrams" from the Eiffel tower in 1959), but initially the attention remained confined to limited groups. In Europe, the field started to develop strongly in the late 1990 s, partially thanks to ESA initiatives. A need for coordination was soon identified, and this led in 2003 to the birth of COST Action $724^{1}$ (2003-2007) on the science of space weather. The Management Committee of COST 724 focused on several ambitious objectives in order to assemble the European Space Weather initiatives and make Europe a major player in the field: (i) to define what is Space Weather in the European perspective, which has specific historical roots, (ii) to stimulate the creation of a European space weather centre, (iii) to open a web portal respectful to the European cultures and (iv) to create a European annual meeting dedicated to space weather. These goals were achieved within the lifetime of COST 724

Following the recommendation by COST 724 we define our field as follows:

Space weather is the physical and phenomenological state of natural space environments. The associated discipline aims, through observation, monitoring, analysis and modeling, at understanding and predicting the state of the sun, the interplanetary and planetary environments, and the solar and non-solar driven perturbations that affect them; and also at forecasting and nowcasting the possible impacts on biological and technological systems.

Space Climate addresses the impact of solar variability on climate and deals with the middle to long term aspects of Space Weather.

During the 4 years of COST 724, independent national activities in Belgium led to the establishment of a European space weather centre under the name "Solar Terrestrial Centre of Excellence" (STCE, http://www.stce.be/). Its birth was strongly supported by the Management Committee of COST 724 and its participants. The European Space Weather Web Portal (ESWeP, http://www.spaceweather.eu/), hosted at the STCE, is one of the outcomes of this collaboration. ESWeP delivers information about space weather data, services, models and research. Besides its scientific content, the ESWeP boasts of an elaborated public outreach section, where information appears in many different European languages.

Another important outcome was the establishment of the European Space Weather Week (ESWW) as the central annual space weather meeting in Europe, which has become more successful every year. Starting with 125 participants in 2004, it

\footnotetext{
${ }^{1}$ Lilensten, J., T. Clark, and A. Belehaki, Europe's first space weather think tank - A new program called COST Action 724 is poised to become the central coordinator of Europe's space weather research initiatives, Space Weather 2 S04001, 2004.
}

doubled this number at its seventh edition in November 2010 (http://sidc.be/esww7).

Towards the end of COST 724, the need for a new journal dedicated to space weather became obvious for the newly established and fast-growing European Space Weather Community. Fortunately, a new action devoted to space weather products and services was soon approved: COST ES0803 ${ }^{2}$ (20082012). The new Action focuses on the development of space weather products and services in Europe (http://www.cos tes0803.noa.gr/). Its Management Committee took new initiatives with great enthusiasm, among them as a primary goal the establishment of the new journal. Contacts were taken with four international scientific publishers. EDP Sciences (http:// publications.edpsciences.org/) was selected. EDPS is world leader in several fields including astronomy and astrophysics.

The Management Committee of COST Action ES0803 decided that the articles should be published with open access. That means that anyone will be allowed to download the papers for free: the authors and sponsors pay for the publication. EDPS and COST accepted to fully cover the publication cost of the first two years. The initial collaborators of the new journal are the COST Office (COST-ESF), the COST Action ES0803 and STCE which provides support for the journal's Editorial Office, a key point for a healthy future of the journal.

Finding a name for the journal was not an easy task. It had to contain the words "space weather" but also reflect new developments in Europe. Over the last two years space climate has become a major concern as a consequence of the lack of knowledge about the mechanisms by which solar activity may force the lower atmosphere meteorology and the climate. This open question is so important that the COST office approved a new Action, ES1005, to address it. ES1005 "Towards a more complete assessment of the impact of solar variability on the Earth's climate" emanated from the research activities of several colleagues already involved in the new journal. Therefore, we decided to accommodate space climate and decided in agreement with our Publisher to name the journal:

"Journal of Space Weather and Space Climate" with acronym SWSC.

SWSC was presented for the first time to the pertinent scientific community during the Seventh European Space Weather Week in Bruges in November 2010 (http://sidc.oma.be/ esww7/). Since then the web site of the journal, http:// www.swsc-journal.org/, is open to accept submissions.

The establishment of a team responsible to act as the task force for the journal content was accomplished in two steps.

${ }^{2}$ Belehaki A., J. Watermann, J. Lilensten, A. Glover, M. Hapgood, M. Messerotti, R. van der Linden, and H. Lundstedt, Renewed Support Dawns in Europe: An Action to Develop Space Weather Products and Services, Space Weather - The International Journal of Research and Applications 7: Art. No. S03001, 2009. 
Firstly, we composed an Editorial Board in charge of the dayto-day tasks of the journal. Then, we created an Advisory Board which involves colleagues from within and outside of Europe including Australia, the United States, Russia, South Africa, China, Egypt and Brazil. Their principal role will be to help us in strategic decisions and in the promotion of SWSC at national levels.

The two Boards worked out the aims and scope of the journal:

The Journal of Space Weather and Space Climate (SWSC) is an international, peer-reviewed, multidisciplinary and interdisciplinary open access journal, which publishes papers on all aspects of space weather and space climate. Coverage includes, but is not limited to:

- fundamental and applied scientific research including theory, observation, data analysis, modelling and prediction

- technical applications and engineering solutions

- impact on human beings and technology in space, in the air, at sea and on land

- societal and economic implications

- educational and dissemination concepts and experiences

- development of user-targeted products and services

- scientific, technical, political and commercial initiatives.

SWSC accepts manuscripts related to space weather and to space climate from a broad range of fields including solar physics, space plasma physics, aeronomy, planetology, radio science, informatics, geophysics, biology, medicine, astronautic, aeronautic and electrical engineering, meteorology, climatology, mathematics and economics.
SWSC publishes regular research articles, short communications, invited reviews, technical and observational reports, strategic and educational articles and concise project reports. All manuscripts are peer reviewed according to the quality standards of international scientific journals. It is therefore required that all manuscripts submitted for publication in SWSC contain enough new insight; present the results against a properly referenced background of existing work; present adequate evidence that supports the conclusions; write clearly, concisely and comprehensively and include all necessary and appropriate figures and tables but not more.

Accepted papers are published in electronic form only.

EDP Sciences maintains an electronic open access archive of all published material.

SWSC will be indexed in Crossref and therefore in all the international Digital Library reference systems.

The kick-off meeting was held in Paris in early October 2010, hosted by EDPS. Major part of the meeting was devoted to the definition of the editorial procedure: all manuscripts are prescreened by the Editors in Chief, and if they pass this filter then they are assigned to an Associate Editor (AE). The AE invites at least two independent experts to review the manuscript. The reviewers are asked to evaluate the paper according to the standards of international peer-reviewed journal, for all types of submissions (regular papers, invited reviews, technical reports, project reports, letters, outreach and educational papers, etc.). There is no limit on the number of revisions, but our aim is to accept a manuscript within 6 months after its first submission.

We thank the authors who dared to submit their excellent work to our journal. We believe that the quality of the first papers published will be the best publicity and attract other excellent submissions. The big adventure has started!!! 